\title{
Dendritic cells recruitment in melanoma metastasis treated by electrochemotherapy
}

\author{
Gianni Gerlini - Serena Sestini • \\ Paola Di Gennaro • Carmelo Urso • \\ Nicola Pimpinelli $\cdot$ Lorenzo Borgognoni
}

Received: 4 November 2011/ Accepted: 13 June 2012

(C) Springer Science+Business Media B.V. 2012

\begin{abstract}
Electrochemotherapy (ECT) is a novel treatment for recurrent or in-transit unresectable melanoma metastases based on the administration of anti-neoplastic drugs followed by cancer cell electroporation. Whether ECT can also induce anti-tumour immunity is unclear. We addressed this issue investigating the presence of dendritic cells (DCs) in the inflammatory infiltrate of ECT-treated lesions. Biopsies from melanoma patients $(n=9)$ were taken before ECT (T0), at d7 and d14 after treatment and studied by immunofluorescence with DCs-related antibodies. Epidermal Langerin $^{+}$Langerhans cells (LCs) were the most represented subset before treatment. ECT induced a significant reduction in epidermal LCs number at $\mathrm{d} 7(p<0.001)$, while they were completely replaced at d14. Similarly, the few LCs observed intermingled with metastatic melanoma cells at T0 decreased after treatment $(p<0.001)$, suggesting an ECTinduced activation of LCs. Consistently, at d1 after ECT ( $n=3$ patients), LCs were found to express CCR7, which mediates LCs migration to regional lymph nodes, and CD83, the typical DCs maturation marker. In contrast, plasmacytoid DCs (pDCs) were not present at $\mathrm{T} 0$, but significantly increased after ECT both in melanoma metastasis
\end{abstract}

G. Gerlini $(\bowtie) \cdot$ S. Sestini · L. Borgognoni

Plastic Surgery Unit, Regional Melanoma Referral Center,

Tuscan Tumour Institute (ITT), Santa Maria Annunziata

Hospital, Via Antella 58, 50012 Bagno a Ripoli, Florence, Italy

e-mail: gianni.gerlini@asf.toscana.it

P. Di Gennaro · N. Pimpinelli

Clinical, Preventive and Oncologic Dermatology Section,

Department Critical Care Medicine and Surgery, University

of Florence, Florence, Italy

C. Urso

Dermatopathology Section, Department Anatomic Pathology,

Santa Maria Annunziata Hospital, Florence, Italy $(p<0.001)$ and perilesionally $(p<0.05)$. Similarly, CD1c $\mathrm{c}^{+}$ dermal DCs (dDCs), observed in low number before ECT, strongly increased at $\mathrm{d} 7$ and even more at $\mathrm{d} 14(p<0.05$ and $p<0.001$, respectively). Notably, some dDCs expressed CD83. These data suggest that ECT promotes LCs migration from the tumour to draining lymph nodes and pDCs and $\mathrm{dDCs}$ recruitment at the site of the lesion. These findings may help to design new strategies of in situ DCs vaccination in cancer patients.

Keywords Dendritic cells · Electrochemotherapy · Melanoma $\cdot$ Immunotherapy

\begin{tabular}{ll}
\multicolumn{2}{l}{ Abberviations } \\
DCs & Dendritic cells \\
dDCs & Dermal dendritic cells \\
ECT & Electrochemotherapy \\
HE & Haematoxylin/eosin \\
IDO & Indoleamine 2-3 dioxigenase \\
LCs & Langerhans cells \\
LN & Lymph node \\
MART-1 & Melanoma antigen recognized by T-cells \\
pDCs & Plasmacyotid dendritic cells \\
TAA & Tumour associated antigens
\end{tabular}

\section{Introduction}

Electrochemotherapy (ECT) is emerging as a highly effective local treatment for skin metastasis of human cancers and it is particularly used for recurrent or in-transit unresectable melanoma metastases [1,2]. ECT treatment is based on the administration of anti-neoplastic drugs, such as bleomycin or cisplatin, followed by the electroporation of the lesions [3]. The electric currents, increasing cell membrane permeability, 
promote the intracellular concentration of the drug and increase its cytotoxicity [4]. Although the overall objective response rate is high, approximately 80-90\% [1], it is generally accepted that ECT has only local effects, therefore it is currently used as a palliative treatment. However, some findings support the view that ECT can also induce anticancer immunity, especially in melanoma which is considered the most immunogenic tumours [5, 6]. Particularly, ECT treatment combined with IL-2 administration induced specific CTL responses [5]. Indeed, melanoma expresses several well known tumour associated antigens (TAA) [6, 7], although most of them are self antigens to which a considerable immunological tolerance is maintained [8]. ECT induces a massive cancer cell death, with the release of several TAA which might become available for immunity activation. For these reasons, melanoma represents an ideal model to study the possible involvement of the immune system after ECT.

Dendritic cells (DCs) are the professional antigen presenting cells with the unique ability to initiate primary immune responses and are supposed to play a key role in cancer immunology $[9,10]$. DCs orchestrate the immune responses and are capable to generate immunity or, in contrast, tolerance towards TAA primarily depending on their maturation state and on the tumour microenvironment $[7,8]$. However, properly stimulated DCs represent a powerful tool to overcome the immune tolerance conditions towards melanoma TAA and generate potent anti-cancer immune responses [8]. Indeed, DC-based immunotherapy has been reported to induce regression of metastatic lesions in melanoma patients [11].

The possibility to exploit the ECT technology aiming to extend the local results to an effective systemic treatment is an intriguing challenge. Thus, the application of ECT followed by DC-based immunotherapy would be a reasonable approach to reach this goal. The release of TAA after ECTinduced melanoma metastasis destruction could represent an ideal source of antigens for local DCs that, upon migration to the draining lymph node (LN), might present these antigens to specific cytotoxic $\mathrm{T}$ cells.

In this study, we analyzed the presence of the different DC subsets at the site of the lesion, before and after ECT treatment, in order to investigate the feasibility of DCbased immunotherapy following ECT treatment.

\section{Methods}

Patients and human tissues

Melanoma patients $(n=9)$ with histological confirmed skin metastasis undergoing ECT with bleomycin were enrolled in the study after obtaining informed written consents. The study was conducted according to Declaration of
Helsinki Principles and Local Institutional Ethics Committee approved all described analyses.

Cutaneous and/or subcutaneous melanoma metastases were treated using Cliniporator ${ }^{\mathrm{TM}}$ device (IGEA Ltd, Modena, Italy). Electric pulses were applied with the appropriate type of electrodes (IGEA Ltd): by needle electrodes (Type II and III) to deeper seated metastasis and by plate electrodes (Type I) to superficial metastasis. The needle electrodes were chosen based on metastasis dimensions: Type II for small nodules (less than $1 \mathrm{~cm}$ ) and Type III for large nodules (more than $1 \mathrm{~cm}$ ). All the lesions treated were smaller than $3 \mathrm{~cm}$ in diameter. Bleomycin was administered intravenously $\left(15,000 \mathrm{UI} / \mathrm{m}^{2}\right.$ body surface area) in a time frame of $60 \mathrm{~s}$. The electrical pulses (variable amplitude with $1-5,000 \mathrm{~Hz}$ delivery frequencies) were applied to the metastasis in a time window between 8 and 28 min after bleomycin infusion to obtain the optimal response utilizing the most appropriate drug concentrations in tissues, according to previous standardized procedure $[1,4]$.

Skin biopsies were obtained before ECT, indicated as T0, and at seven and 14 days after treatment, indicated as $\mathrm{d} 7$ and $\mathrm{d} 14$ post, from different lesions. In the last three patients enrolled in the study we obtained additional samples after $24 \mathrm{~h}$, indicated as $\mathrm{d} 1$ post. Specimens were embedded in OCT-like medium (Killik; Bio-Optika, Milan, Italy), snapfrozen in liquid nitrogen and stored at $-80{ }^{\circ} \mathrm{C}$. Cryostat sections $(10-12 \mu \mathrm{m})$ were fixed for $10 \mathrm{~min}$ in cold acetone (Sigma, Milan, Italy) at $4{ }^{\circ} \mathrm{C}$, air dried at room temperature and stored at $-20^{\circ} \mathrm{C}$. Skin biopsies were also processed for routine histology with haematoxylin/eosin (HE).

\section{Antibodies}

The following mouse anti-human antibodies were used: CD83 (HB15e, IgG1), CD86 (2331 FUN-1, IgG1) and HLA-DR (L243, IgG2a), FITC-conjugated, from BD-Pharmingen (San Diego, CA); BDCA-2 (AC144, IgG1), FITC-conjugated, from Miltenyi Biotec (Bergisch Gladbach, Germany); Langerin (CD207; DCGM4, IgG1) and CD1c (L161, IgG1), from Immunotech (Marseille, France); CD1a-FITC (NA1/34, IgG2a), from DakoCytomation (Glostrup, Denmark); MART1/Melan A (A103, IgG1), from Ventana Medical System, Inc. (Tucson, AZ); CCR7 (6B3, IgG1), from MBL International Corporation (Woburn, MA). Goat anti-mouse Alexa Fluor 594 was used as secondary antibodies and fluorescein signal was amplified with anti FITC Alexa Fluor 488 conjugated, both from Molecular Probes (Invitrogen, Milan, Italy). Isotype-matched antibodies were used as negative controls.

Immunofluorescence

Double immunofluorescence analyses were performed on cryostat sections at room temperature unless otherwise 
indicated. Sections were pre-treated with $20 \mathrm{mg} / \mathrm{ml} \mathrm{BSA} \mathrm{in}$ PBS (Sigma) for $2 \mathrm{~h}$ and stained first with un-conjugated antibodies for $2 \mathrm{~h}$, revealed with anti mouse Alexa Fluor 594 secondary antibodies, then with FITC-conjugated antibodies, overnight at $4{ }^{\circ} \mathrm{C}$; fluorescein signal was amplified with anti FITC Alexa Fluor 488 antibodies. Nuclei were labelled with Hoechst $33342(20 \mu \mathrm{g} / \mathrm{mL}$; Sigma). Slides were mounted with Prolong antiFade (Invitrogen) and observed with Leica DMR HC microscope equipped for epifluorescence (Leica Microsystems GmbH, Wetzlar, Germany).

Image acquisition and analysis

Images were acquired at 6.64 and $0.529 \mu \mathrm{m}$ per pixel resolution, corresponding to $16 \times$ and $200 \times$ original magnifications respectively, using Leica DC200 microscope digital colour camera and Leica DC Viewer software. For each specimen, $n=5$ microscopic non-consecutive fields were acquired at $200 \times$, examined for quantitative analyses by two independent observers (P.D.G. and G.G.), and the average number of positive cells was determined. Then the mean number of positive cells from all the melanoma samples ( $n=9)$, at different time-points (T0, d7, d14), was calculated. Quantitative analyses were first performed by counting DCs defined as HLA-DR ${ }^{+}$large mononuclear cells with dendrities, as previously described [12]. Then quantitative analyses were performed by counting directly the different DC subsets by using specific monoclonal antibodies, thus Langerhans cells (LCs) were identified as Langerin $^{+}$and $\mathrm{CD} 1 \mathrm{a}^{+}$cells, dermal DCs (dDCs) as CD1c ${ }^{+}$ $\mathrm{HLADR}^{+}$cells and plasmacytoid DCs (pDCs) as BDCA2 ${ }^{+}$ cells.

\section{Statistical analysis}

The values reported throughout the text are expressed as mean \pm SD and represent the mean number of positive cells from all the patients $(n=9)$, at different time-points (T0, d7, d14).

Statistical evaluation was performed using two-sided Student's $t$ test with $p<0.05$ adopted as the level of significance.

\section{Results}

Clinical effects of electrochemotherapy on metastatic melanoma lesions

In order to establish the appropriate time to take biopsies from ECT-treated lesions, we first addressed our attention on the clinical signs of inflammation following the treatment. Metastatic lesions usually appeared as a well delimited nodule either elevated on the skin surface (Fig. 1a, upper panels) or localized in the subcutaneous tissue (Fig. 1a lower panels, b, and c). Few hours after ECT treatment, the lesions appeared swollen and reddish due to an inflammatory reaction that gradually increased until d714 after treatment in parallel with a marked regression of the lesions (Fig. 1a). Both cutaneous and subcutaneous lesions responded to ECT. Based on these clinical observations, we decided to take biopsies after seven (d7) and 14 days (d14) from treatment. The biopsies were performed at the margin of the lesion to include a fragment of metastasis and a rim of apparently healthy perilesional tissue (Fig. 1b, c). In place of HE staining (Fig. 1b), the different anatomical compartments were identified throughout the study by Hoechst staining (Fig. 1c), a nuclear fluorescent dye (blue). Metastatic cells were identified by the expression of MART-1 (Melanoma Antigen Recognized by T-cells), a typical protein of melanocyte lineage and used for melanoma diagnosis.

Cutaneous DC subsets within metastatic melanoma lesion before electrochemotherapy

Before treatment (T0) Langerhans cells (LCs), detected by the co-expression of CD1a and Langerin [13], were the most represented skin DC subset, located in the epidermis over the tumour mass and rarely scattered in the dermis (Fig. 2a). Epidermal LCs expressed HLA-DR (Fig. 2b), little amount of CD86, but not CD80 and CD83 (data not shown), exhibiting a typical immature immunophenotype. A distinct subpopulation of Langerin ${ }^{-} \mathrm{HLA}_{-} \mathrm{DR}^{+}$dendritic appearing cells was also observed scattered in the upper dermis and in peritumoural dermis (Fig. 2b, c). A further characterization showed that most of these Langerin ${ }^{-}$ HLA-DR $^{+}$cells in the dermis co-expressed the CD1c antigen, a marker of dermal DCs (dDCs) [14] (Fig. 2d). Few LCs were also detected within the tumour mass, strongly positive for MART-1 (Fig. 2e). Plasmacytoid DCs (pDCs), the lymphoid DC subset identified by the specific expression of BDCA-2 antigen [15], were not observed before treatment (data not shown). Thus, in metastatic lesions, the DCs compartment at T0 is mainly represented by epidermal LCs and, at lesser extent, by dDCs.

Epidermal langerhans cells activation after electrochemotherapy

After treatment a significant reduction of epidermal LCs number was observed at $\mathrm{d} 7$ as compared to $\mathrm{T} 0$ (3.01 \pm 0.88 and $14.98 \pm 2.41$, respectively; $p<0.001$ ) (Fig. 3a, c). Importantly, in some cases LCs were 

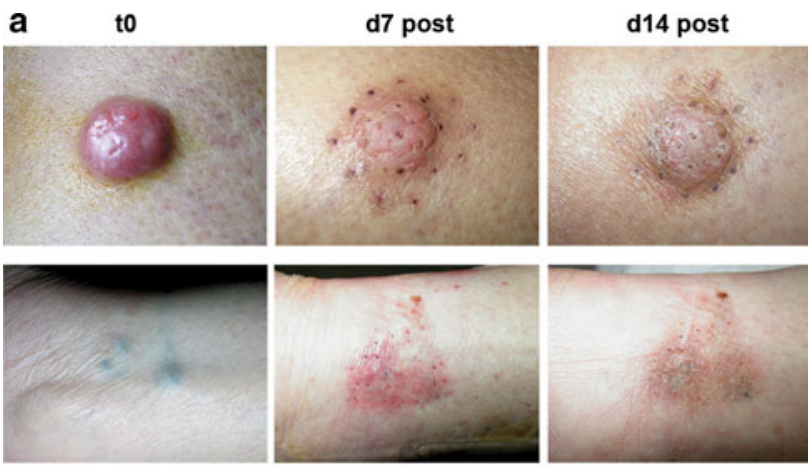

b

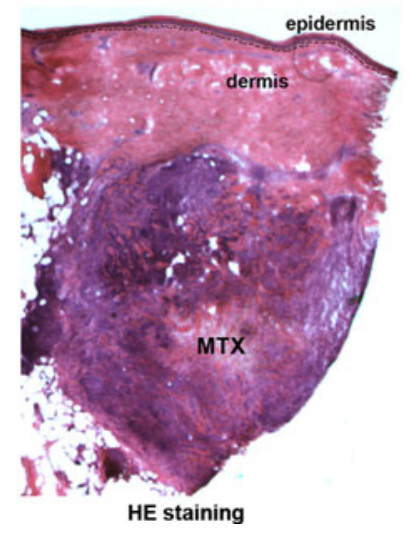

\begin{abstract}
c
\end{abstract}

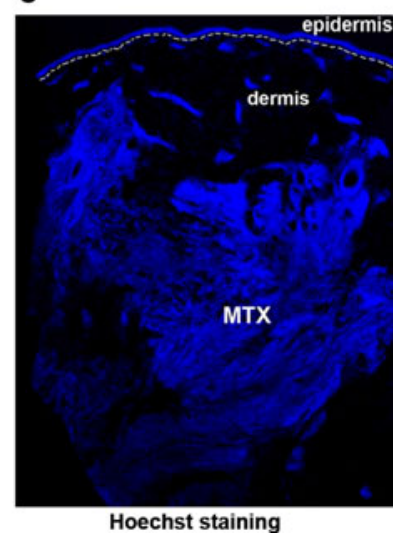

Fig. 1 Skin melanoma metastatic lesions before and after ECT treatment. a Two typical melanoma metastases; images before ECT (T0) and 7 and 14 days after treatment (d7 and d14 post). An exophytic cutaneous (upper panels) and subcutaneous (lower panels) metastases are shown. $\mathbf{b}-\mathbf{c}$ Representative serial frozen sections from a subcutaneous metastasis, labelled with standard haematoxylin and eosin (HE) (b) and with Hoechst (c), a nuclear fluorescent dye (blue), used here as surrogate of haematoxylin nuclear staining to compare the different tumoural and peritumoural anatomical compartments: the epidermis, the dermis, and the metastatic nodule (MTX). Dotted lines indicate the epidermal-dermal barrier (Original magnification $\times 16$ ). (Color figure online)

completely disappeared from the epidermis while the few cells observed displayed a round morphology, apparently without dendritic processes (data not shown). These data suggested that ECT might have induced a rapid LCs migration to the local draining LNs. In order to investigate this issue, we took additional biopsies at $\mathrm{d} 1$ after ECT ( $n=3$ patients) to analyse the expression of CD83, the DC maturation marker, and CCR7, which mediates LC migration to regional LNs [16]. Indeed at $\mathrm{d} 1$ after ECT some epidermal LCs expressed CD83 (Fig. 3d) and CCR7 (Fig. 3e). Moreover, we observed a reduction in the LCs number, as compared to $\mathrm{T} 0$, although not significant.

Interestingly on d14 the epidermal LCs subset, strongly Langerin $^{+}$HLA-DR $^{+}$and with the typical dendritic appearance, was almost reconstituted and similar to T0 (d14: $14.01 \pm 3.19)$ (Fig. 3b, c). a

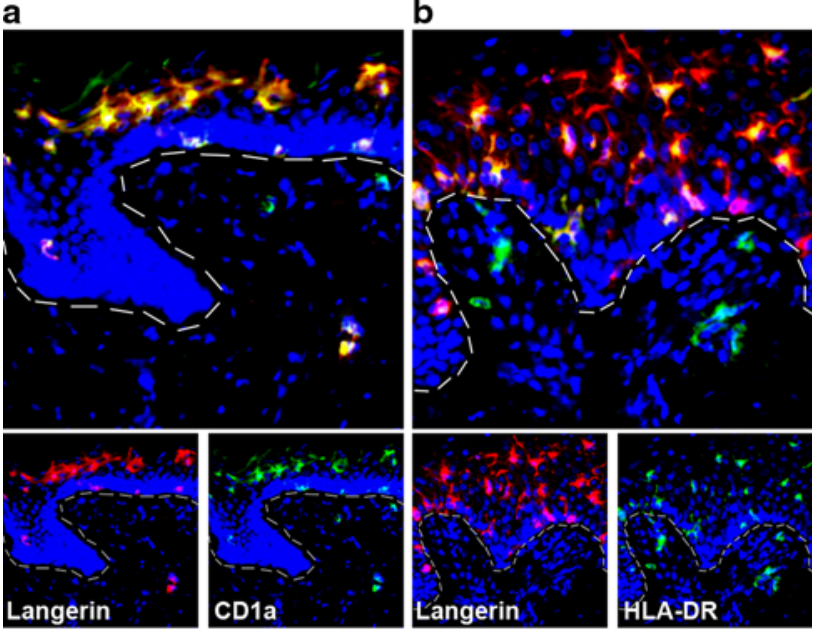

c

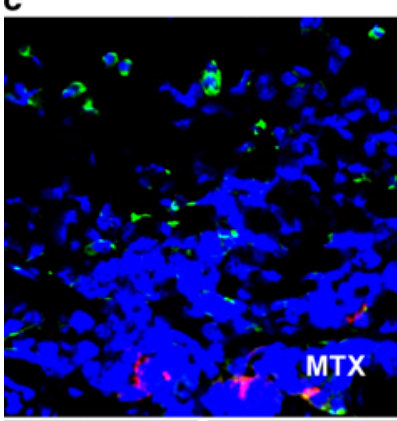

d

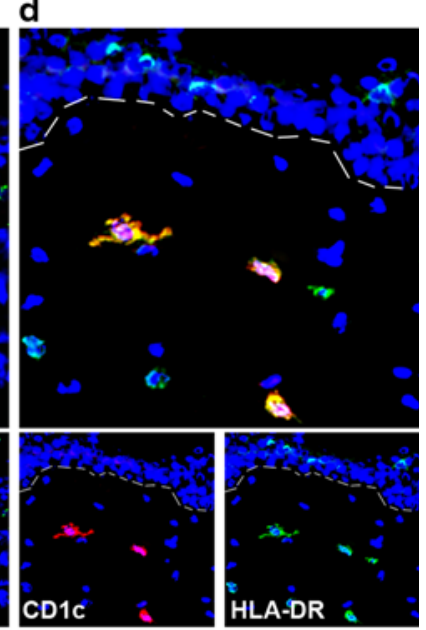

Langerin

e

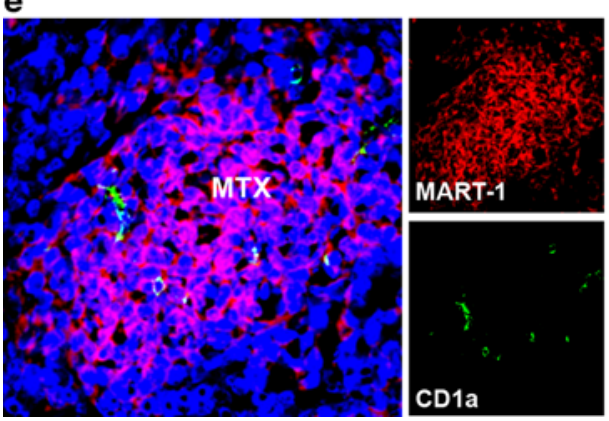

Dermal and plasmacytoid DCs recruitment in the perilesional dermis after electrochemotherapy

In the dermis the number of Langerin ${ }^{+}$LCs did not significantly vary from $\mathrm{T} 0$ to $\mathrm{d} 7$ and $\mathrm{d} 14$ after treatment $(1.35 \pm 0.49,1.65 \pm 0.89$ and $1.9 \pm 0.8$, respectively) (Fig. 4d). However, in line with clinical observations, the inflammatory infiltrate in the dermis, that was modest at T0, increased considerably after treatment (data not shown). In particular a high number of Langerin ${ }^{-}$HLA$\mathrm{DR}^{+}$dendritic appearing cells infiltrated the peritumoural 
4Fig. 2 Skin DC subsets before ECT treatment Double immunofluorescence analyses on frozen sections of skin melanoma metastases $(n=9)$ before ECT treatment were performed with the indicated antibodies, revealed with Alexa Fluor 594 (red) and 488 (green), respectively. Co-expression of antigens is indicated as merge (yellow/ orange). Nuclei were labelled with Hoechst dye (blue). Details from representative images are shown. a Langerin (red) and CD1a (green) double staining identifies epidermal LCs (yellow/orange). b Langerin (red) and HLA-DR (green) double staining shows co-expression on epidermal LCs (yellow/orange); some Langerin ${ }^{-} \mathrm{HLA}^{-\mathrm{DR}^{+}}$cells (green) are observed in the upper dermis. $\mathbf{c}$ The Langerin ${ }^{-}$HLA-DR ${ }^{+}$ cell population (green) is present in the peritumoural dermis, while Langerin $^{+}$HLA-DR ${ }^{+-}$LCs (orange) are observed within the tumour mass. d CD1c (red) and HLA-DR (green) double staining revealed that HLA-DR ${ }^{+}$dendritic cells co-expressed CD1c (yellow/orange). e MART-1 (red) and CD1a (green) double staining shows the presence of $\mathrm{CD}^{+} \mathrm{a}^{+} \mathrm{LCs}$ within the tumour mass, characterized by strong expression of MART-1 (Original magnification $\times 200$ ). (Color figure online)

dermis at d7 and increased at d14 (Fig. 4a, b). These cells were mainly located around the shrinking tumour mass and they were often very close to melanoma cells (Fig. 4a, b). We quantified the HLA-DR ${ }^{+}$dendritic appearing cells at $\mathrm{T} 0, \mathrm{~d} 7$ and $\mathrm{d} 14$ post. The mean number of HLA-DR ${ }^{+}$DCs significantly increased from $\mathrm{d} 7(12.46 \pm 1.94)$ to $\mathrm{d} 14$ $(23.14 \pm 5.71)$ as compared to T0 $(8.35 \pm 1.24)(p<0.05$ and $p<0.01$, respectively) (Fig. 4c). A further immunophenotype characterization of this HLA-DR ${ }^{+}$DCs population revealed that the majority co-expressed CD1c thus identifying these cells as dDCs (Fig. 4e, f). The number of CD1c ${ }^{+}$dDCs significantly increased from $\mathrm{d} 7$ (8.34 \pm $1.69)$ to $\mathrm{d} 14(16.11 \pm 2.95)$ after ECT as compared to T0 $(5.63 \pm 1.01) \quad(p<0.05$ and $p<0.001$, respectively $)$ (Fig. 4d). On d7 and even more on d14 some of CD1c ${ }^{+}$ DCs were positive for CD83 (Fig. 4g, h), suggesting that $\mathrm{dDCs}$ reached the maturation at peritumoural level.

Importantly, the inflammatory infiltrate observed in the perilesional dermis contained the BDCA2 ${ }^{+}$pDCs, a DCs subset not detected at $\mathrm{T} 0$, which significantly increased from $\mathrm{d} 7$ to $\mathrm{d} 14(4.37 \pm 1.49$ and $7.9 \pm 2.58$, respectively; $p<0.05$ ) (Fig. 4d).

DC subsets within metastatic melanoma cells after electrochemotherapy

At $\mathrm{d} 7 \mathrm{pDCs}$ were also observed within the metastatic cells $(7.5 \pm 2.08)$ (Fig. 5a, e) and the number significantly increased at $\mathrm{d} 14$ post ECT $(15.66 \pm 1.74, p<0.001)$, when pDCs were typically observed in clusters around and within the melanoma mass (Fig. 5b, e). In contrast, the few intra-tumoural CD1a ${ }^{+} \mathrm{LCs}$ appeared reduced in number on d7 as compared to T0 $(1.25 \pm 0.47$ and $4.41 \pm 0.9$, respectively; $p<0.001$ ) (Fig. 5c, e) and were not observed at d14 after ECT (Fig. 5d, e). Notably, while expression of MART-1 was homogeneous at T0 (Fig. 2e), it became
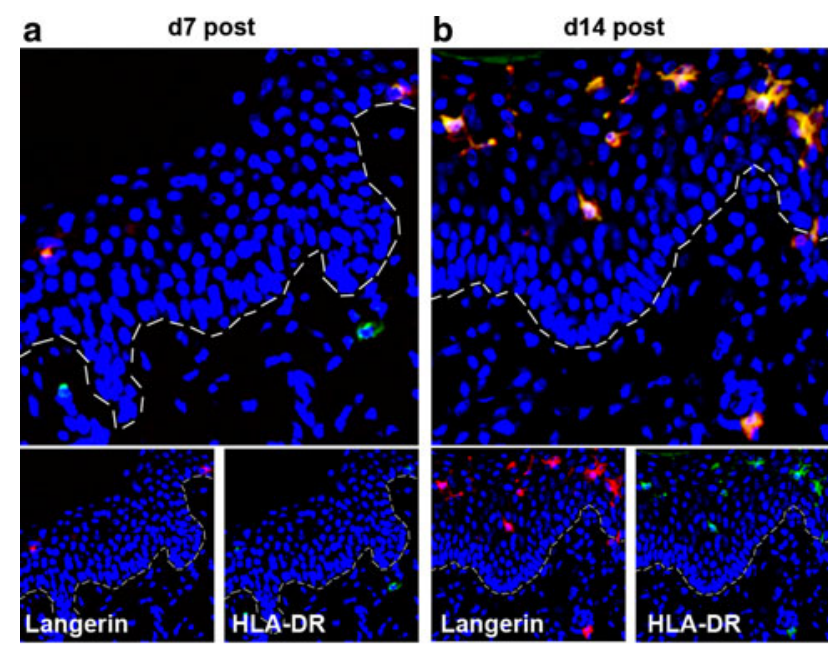

Langerin

HLA-DR
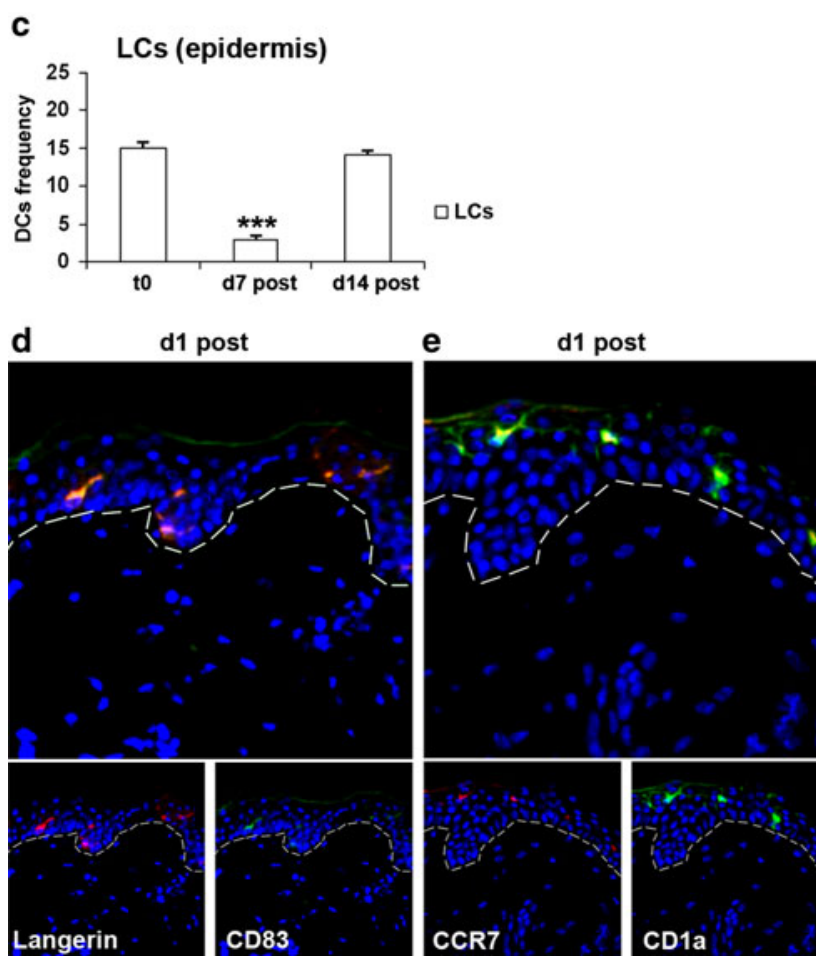

Fig. 3 Epidermal LCs after ECT treatment Double immunfluorescence analyses on frozen sections from ECT-treated melanoma metastases $(n=9)$ were performed with the indicated antibodies as described in legend for Fig. 2. Details from representative images are shown. a Epidermal Langerin ${ }^{+}$HLA-DR $^{+-}$(orange) LCs were reduced in number at d7 from ECT. b At d14 from ECT, LCs were well represented within the epidermis and co-expressed Langerin and HLA-DR (yellow/orange). c For quantitative analyses the number of positive cells for each specimen was assessed by calculating the average cell number counted in $n=5$ different fields. Values represent the mean number of positive cells from $n=9$ melanoma samples. Triple asterisks indicates $p<0.001$. Epidermal LCs analyses show a significant reduction of LCs number at $\mathrm{d} 7$ from ECT and a subsequent repopulation at $\mathrm{d} 14$. d-e At d1 after ECT, some Langerin $^{+}$LCs co-expressed CD83 (yellow/orange; d) and CCR7 (yellow/orange; e) within the epidermis ( $n=3$ patients) (Original magnification $\times 200)$. (Color figure online) 

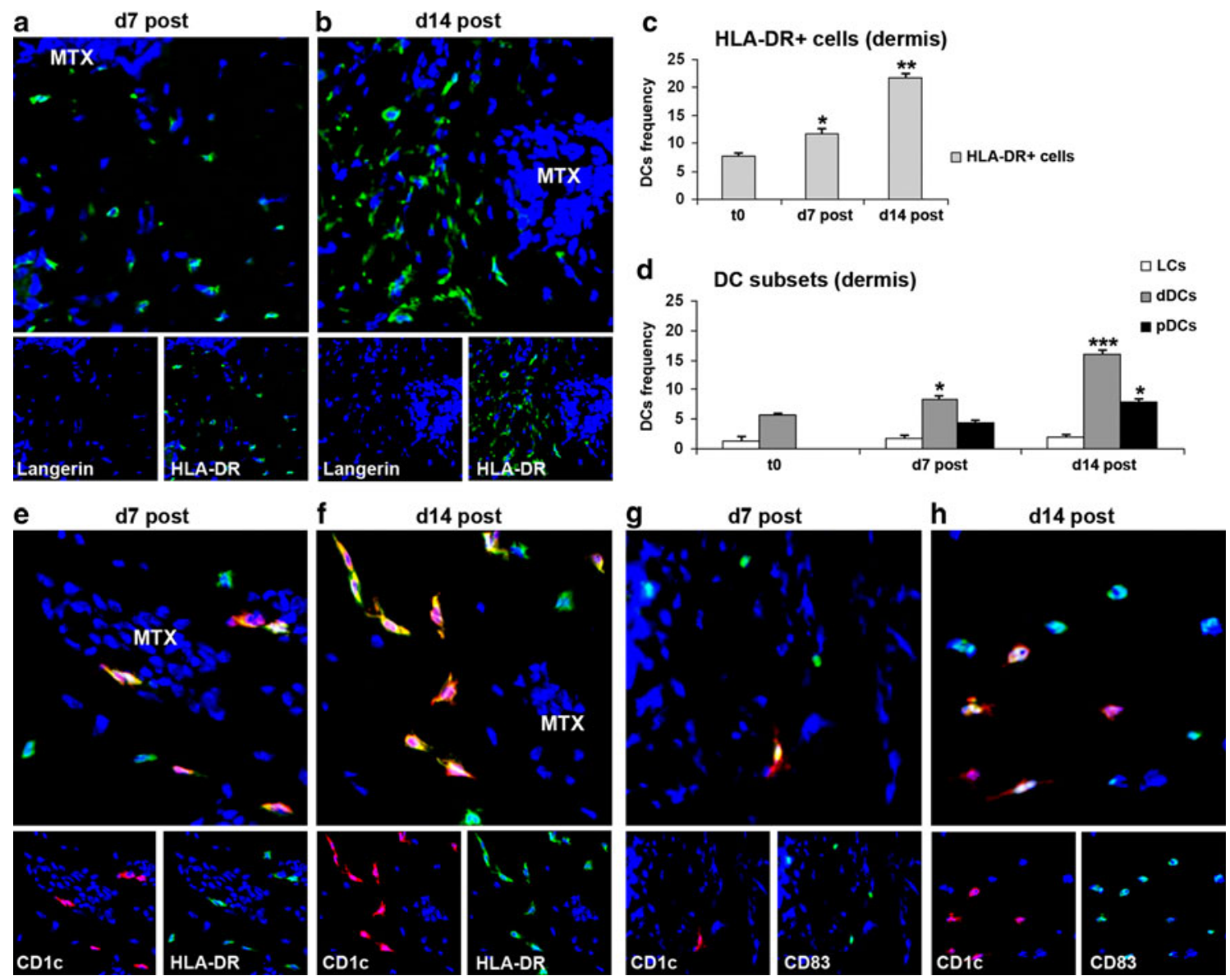

Fig. 4 DC subsets in the dermis after ECT treatment Double immunfluorescence analyses on frozen sections from ECT-treated melanoma metastases $(n=9)$ were performed with the indicated antibodies as described in legend for Fig. 2. Details from representative images are shown. a-b At d7 from ECT, a high number of dermal peritumoural Langerin ${ }^{-} \mathrm{HLA}^{-\mathrm{DR}^{+}}$dendritic looking cells (green) were observed (a), which further increased at d14 (b). c-d Quantitative analyses were performed as described in legend for Fig. 3; values represented the mean frequency of positive cells per digital image. Asterisks indicates $p<0.05$; double asterisks indicates $p<0.01$ and triple asterisks indicates $p<0.001$. c HLA-DR ${ }^{+}$cells

extremely variable at $\mathrm{d} 7$ and $\mathrm{d} 14$ post ECT, probably due to the different grade and speed of tumour cell death (Fig. 5a-d). The persistence of MART-1 expression until d14 post ECT, despite of clinical tumour regression, indicates that the source of melanoma TAA are still available for recruited DCs.

\section{Discussion}

It has been shown that ECT induces a recruitment of mononuclear leucocytes within and around the treated metastatic lesions [17]. While the nature of these infiltrate in mice has been unravelled [18], in humans it has not been investigated. Here we show the presence of different DC

analyses showed a significant increase at $\mathrm{d} 7$ and d14 from ECT. d DC subsets analyses in the dermis showed no significant differences in LCs number, a significant increase of dDCs number at $\mathrm{d} 7$ and $\mathrm{d} 14$ from ECT and the appearance of pDCs at $\mathrm{d} 7$ that significantly increase at d14 after treatment. e-f CD1c (red) and HLA-DR (green) double staining revealed that $\mathrm{HLA}-\mathrm{DR}^{+}$dendritic cells co-expressed $\mathrm{CD} 1 \mathrm{c}$ (yellow/orange) (e) and the number of CD1c ${ }^{+} \mathrm{HLA}^{-\mathrm{DR}^{+}} \mathrm{DCs}$ at $\mathrm{d} 14$ from ECT increased (f). $\mathbf{g}-\mathbf{h}$ Some $\mathrm{CD} 83^{+}$cells (green) are observed in the dermis at $\mathrm{d} 7$ from ECT $(\mathbf{g})$, and increased at d14 (h); some of these cells co-expressed $\mathrm{CD}_{\mathrm{c}}{ }^{+}$(yellow/orange) $(\mathbf{g}-\mathbf{h})$ (Original magnification $\times 200)$. (Color figure online)

subsets in the inflammatory reaction that infiltrates ECTtreated skin melanoma metastasis and these results were similar in both cutaneous and subcutaneous nodules. These findings are in line with previous data obtained in a mouse model where it was demonstrated the recruitment of CD11c ${ }^{+}$DCs in ECT-treated metastasis [18], although the effects of the treatment were more rapid than in humans.

Epidermal LCs, the most represented subset before ECT, resulted particularly affected by the treatment which induced a considerable reduction of their number at $\mathrm{d} 7$ and in some cases they almost disappeared from the epidermis. Similarly, the few LCs observed within metastatic cells and in the surrounding dermis were drastically reduced in number at d7. In contrast, after 14 days, LCs normally repopulated the epidermis, displaying their characteristic 

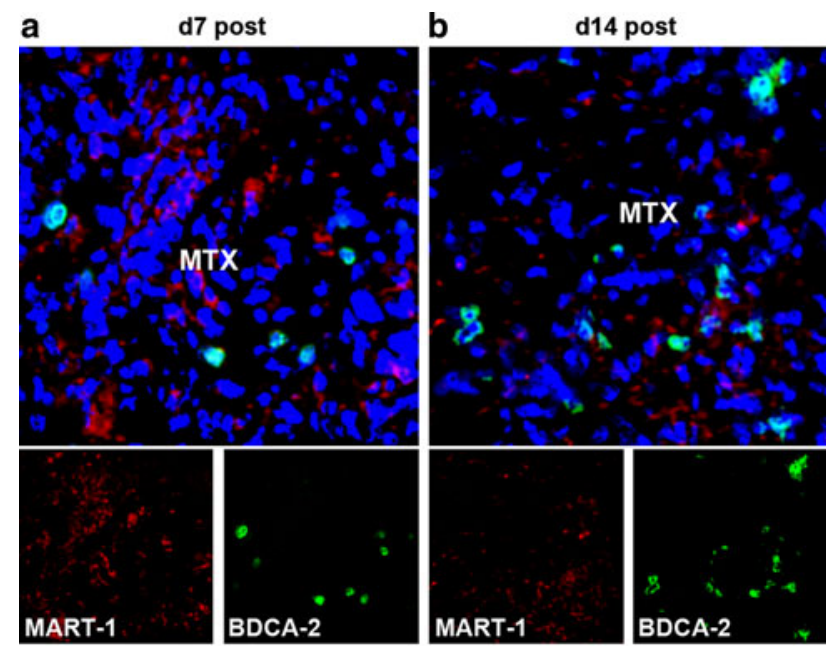

c

d7 post

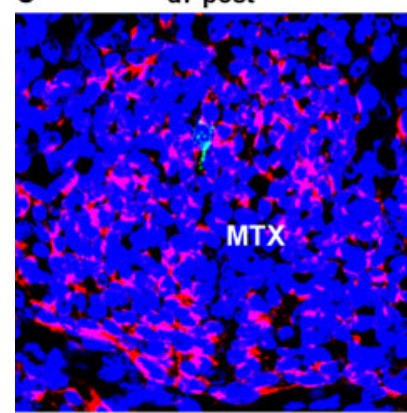

d

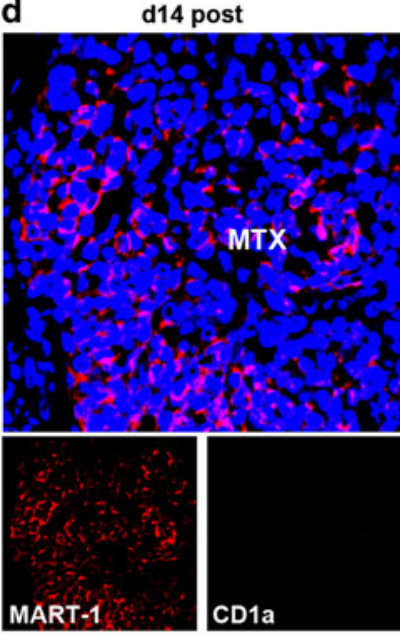

MART-1

CD1a

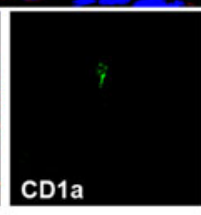

e DCs (MTX)

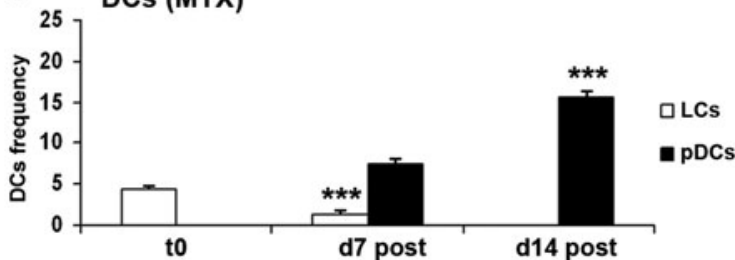

Fig. 5 Intra-tumoural pDCs and LCs after ECT treatment Double immunfluorescence analyses on frozen sections from ECT-treated melanoma metastases $(n=9)$ were performed with the indicated antibodies as described in legend for Fig. 2. Details from representative images are shown. a At $\mathrm{d} 7$ post ECT, scattered BDCA $2^{+} \mathrm{pDCs}$ (green) were observed within the MART- $1^{+}$tumour nests $(\mathrm{red})$. b At d14, $\mathrm{BDCA}^{+} \mathrm{pDCs}$ further increased and were observed in cluster particularly within the MART $-1^{+}$tumour mass. c-d CD1 ${ }^{+}$LCs (green) within the tumour mass decreased in number at $\mathrm{d} 7$ (c) and disappeared at d14 from ECT $(\mathbf{d})$. Note the different expression of MART-1 in panels $(\mathbf{a}-\mathbf{b})$ compares to panels (c-d). e Quantitative analyses of DC subsets within the tumour mass were performed as indicated in Fig. 3. Values represented the mean frequency of positive cells per digital image. Triple asterisks indicates $p<0.001$. DC subsets analyses showed the significant reduction of LCs number within the tumour mass at $\mathrm{d} 7$ and their absence at $\mathrm{d} 14$ from $\mathrm{ECT}$, and the appearance of $\mathrm{pDCs}$ within the tumour mass at $\mathrm{d} 7$ and the significant increase of pDCs number at d14 from ECT (Original magnification $\times 200$ ). (Color figure online) dendritic morphology. These observations suggest that ECT treatment might have stimulated LCs migration to the draining LNs and that this process had occurred in an early phase. To test this hypothesis we performed additional biopsies as early as $24 \mathrm{~h}$ post ECT and studied DCs activation markers. Indeed at this time some epidermal LCs expressed CD83, the classical DC maturation marker, and CCR7, the receptor for CCL20/MIP3b chemokine, which mediates migration to lymphatic vessels [16], in line with the concept that migration is linked to maturation $[19,20]$. Moreover, we observed a reduction in the LCs number, although not significant, suggesting that epidermal LCs had been activated, but most of the cells had still to migrate. These observations are in line with previous report showing that electroporation treatment alone is able to induce migration of murine epidermal LCs into the medium from cultured skin explants, after 24 and $48 \mathrm{~h}$ from treatment [21]. Although we cannot rule out the possibility that some LCs might have died after the treatment due to bleomycin cytotoxic effects enhanced by electrical pulses, it has been reported that ECT does not affect non-proliferating cells [3].

The presence of a normal epidermal LCs population at d14 is consistent with a renewal of LC pool originating from blood precursors.

Interestingly pDCs displayed an opposite behaviour. They were not observed at $\mathrm{T} 0$, but appeared in the peritumoural dermis and within metastatic cells at $\mathrm{d} 7$, and further increased at d14. Although pDCs are able to produce large amount of type I IFN in response to viral infections [22], their role in cancer immunology is controversial. In melanoma patients they are suspected to play a tolerogenic role $[23,24]$ and, consistently, in melanoma draining $\mathrm{LN}$ they express indoleamine 2-3 dioxigenase (IDO), a potent tolerogenic enzyme [25]. However, upon stimulation, in vitro pDCs secrete high level of type I IFN that promote cytotoxic $\mathrm{T}$ cell responses and are capable to prime melanoma specific $\mathrm{CD} 8^{+} \mathrm{T}$ cells $[23,26]$. Interestingly, pDCs increase in several inflamed skin conditions [24]. The presence of pDCs in the inflammatory infiltrate of ECT-treated melanoma metastasis is a key point. These cells express TLR7 and TLR9 therefore their presence represents a prerequisite for the administration of TLR7 and TLR9 agonists, two potent immune stimulators [27], after ECT.

A central role seems to be played by $\mathrm{dDCs}$, which were present at $\mathrm{T} 0$ as scattered $\mathrm{CD} 1 \mathrm{c}^{+} \mathrm{HLA}^{-\mathrm{DR}^{+}}$cells, and strongly increased after ECT treatment reaching the highest concentration at $\mathrm{d} 14$. Of note $\mathrm{dDCs}$ were typically observed around and close to the regressing metastatic melanoma suggesting a key role in capturing TAA that are released by tumour cells death. As already observed for LCs, also dDCs after ECT treatment were found to express 
CD83, which indicates DCs maturation. These observations indicate that ECT induces an important recruitment of dDCs at the site of the treated lesion and some of these dDCs might undergo in situ maturation likely due to high levels of inflammatory cytokines.

Bleomycin may play a key role in DCs recruitment and activation observed in ECT treated lesions. Indeed, in a mouse model, intra-tracheal administration of bleomycin has been reported to induce an increased numbers of mature DCs expressing CD40, CD83 and CD86 [28]. Notably, these lung DCs are considered important in sustaining pulmonary inflammation and fibrosis, a side effect that can also occur in patients undergoing ECT with systemic bleomycin [1, 4]. Even though bleomycin may strongly contribute to DCs recruitment in the perilesional tissue, this result requires some days in mice, in which treatment effects are more rapid than in humans $[18,28]$. Thus it is likely that the rapid expression of CD83 and CCR7 that we observed on LCs at 1d post ECT, might be due to the combination of bleomicyn and the electric pulses.

Although the presence of a high number of DCs and melanoma antigens in the ECT-treated lesion are important requirements for generating anticancer immunity, the clinical observations demonstrate that it is not sufficient. Indeed regression of untreated distant metastases have never been reported and ECT has shown only a local effect [1-3]. This may be due to defective function of peritumoural DCs. Indeed melanoma associated DCs have been shown to be mediators of tumour-induced tolerance in metastatic lesions due to immunosuppressive cytokines $[12,26,29,30]$. However, ECT treatment through the massive cancer cell death and the inflammatory reaction that follows, might alter the melanoma induced immunosuppressive microenvironment [4]. The presence of some $\mathrm{CD}^{+} 3^{+}$DCs supports this hypothesis, nevertheless these DCs are still inadequate to induce a relevant response. In contrast, the administration of immunoadjuvant, such as TLRs agonists, which have been shown to potentiate the level of DC activation, can enhance anti melanoma immunity and achieve a systemic clinical response [18, 32]. In particular, the administration of TLR7 and TLR9 agonists might be important to activate recruited pDCs [27].

In conclusion the high number of DCs and the characterization of the distinct DC subsets in the inflammatory infiltrate of ECT-treated melanoma metastasis provide the rationale for a new therapeutic approach in metastatic melanoma patients by combining ECT treatment with in situ DCs activation and targeting (i.e. in situ DCs vaccination) [31-33]. In this way the effective local effects of ECT might be enhanced and turned in a systemic treatment by the activation of local recruited DCs.
Acknowledgments This work was supported by Ente Cassa di Risparmio di Firenze (Grant number 2010.1392).

Conflict of interest The authors declare that they have no conflict of interest.

\section{References}

1. Marty M, Sersa G, Garbay JR, Gehl J, Collins CG, Snoj M, Billard V, Geertsen PF, Larkin JO, Miklavcic D, Pavlovic I, Paulin-Kosir SM, Cemazar M, Morsli N, Soden DM, Rudolf Z, Robert C, O'Sullivan GC, Mir LM (2006) Electrochemotherapy-An easy, highly effective and safe treatment of cutaneous and subcutaneous metastases: Results of ESOPE (European standard operating procedures of electrochemotherapy) study. Eur J Cancer Suppl 4:3-13

2. Möller MG, Salwa S, Soden DM, O'Sullivan GC (2009) Electrochemotherapy as an adjunct or alternative to other treatments for unresectable or in-transit melanoma. Expert Rev Anticancer Ther 9:1611-1630

3. Mir LM (2006) Basis and rationale of the electrochemotherapy. Eur J Cancer Suppl 4:38-44

4. Mir LM, Gehl J, Sersa G, Collins CG, Garbay J-R, Billard V, Geertsen PF, Rudolf Z, O’Sullivan GC, Marty M (2006) Standard operating procedures of the electrochemotherapy: instructions for the use of bleomycin or cisplatin administered either systemically or locally and electric pulses delivered by the cliniporator by means of invasive or non-invasive electrodes. Eur J Cancer Suppl 4:14-25

5. Andersen MH, Gehl J, Reker S, Pedersen LØ, Becker JC, Geertsen P, Thor Straten P (2003) Dynamic changes of specific T cell responses to melanoma correlate with IL-2 administration. Semin Cancer Biol 13:449-459

6. Renkvist N, Castelli C, Robbins PF, Parmiani G (2001) A listing of human tumour antigens recognized by $\mathrm{T}$ cells. Cancer Immunol Immunother 50:3-15

7. Parmiani G, Castelli C, Dalerba P, Mortarini R, Rivoltini L, Marincola FM, Anichini A (2002) Cancer immunotherapy with peptide-based vaccines: what have we achieved? Where are we going? J Natl Cancer Inst 94:805-818

8. Pardoll DM (1998) Cancer vaccines. Nat Med 4:525-531

9. Cella M, Sallusto F, Lanzavecchia A (1997) Origin, maturation and antigen presenting function of dendritic cells. Curr Opin Immunol 9:10-16

10. Banchereau J, Steinman RM (1998) Dendritic cells and the control of immunity. Nature 392:245-252

11. Nestle FO (2000) Dendritic cell vaccination for cancer therapy. Oncogene 19:6673-6679

12. Gerlini G, Tun-Kuy A, Dudli C, Burg G, Pimpinelli N, Nestle FO (2004) Metastatic melanoma secreted IL-10 down-regulates CD1 molecules on Dendritic cells in metastatic tumour lesions. Am J Pathol 165:1853-1863

13. Valladeau J, Duvert-Frances V, Pin JJ, Dezutter-Dambuyant C, Vincent C, Massacrier C, Vincent J, Yoneda K, Banchereau J, Caux C, Davoust J, Saeland S (1999) The monoclonal antibody DCGM4 recognizes langerin, a protein specific of langerhans cells, and is rapidly internalized from the cell surface. Eur $\mathbf{J}$ Immunol 29:2695-2704

14. Nestle FO, Zheng XG, Thompson CB, Turka LA, Nickoloff BJ (1993) Characterization of dermal dendritic cells obtained from normal human skin reveals phenotypic and functionally distinctive subsets. J Immunol 151:6535-6545 
15. Dzionek A, Fuchs A, Schmidt P, Cremer S, Zysk M, Miltenyi S, Buck DW, Schmitz J (2000) BDCA-2, BDCA-3, and BDCA-4: three markers for distinct subsets of dendritic cells in human peripheral blood. J Immunol 165:6037-6046

16. Yoshida R, Imai T, Hieshima K, Kusuda J, Baba M, Kitaura M, Nishimura M, Kakizaki M, Nomiyama H, Yoshie O (1997) Molecular cloning of a novel human CC chemokine EBI1-ligand chemokine that is a specific functional ligand for EBI1, CCR7. J Biol Chem 272:13803-13809

17. Mekid H, Tounekti O, Spatz A, Cemazar M, El Kebir FZ, Mir LM (2003) In vivo evolution of tumour cells after the generation of double-strand DNA breaks. Br J Cancer 88:1763-1771

18. Roux S, Bernat C, Al-Sakere B, Ghiringhelli F, Opolon P, Carpentier AF, Zitvogel L, Mir LM, Robert C (2008) Tumour destruction using electrochemotherapy followed by $\mathrm{CpG}$ oligodeoxynucleotide injection induces distant tumour responses. Cancer Immunol Immunother 57:1291-1300

19. Wang B, Amerio P, Sauder DN (1999) Role of cytokines in epidermal langerhans cell migration. J Leukoc Biol 66:33-39

20. Bennaceur K, Popa I, Portoukalian J, Berthier-Vergnes O, Péguet-Navarro J (2006) Melanoma-derived gangliosides impair migratory and antigen-presenting function of human epidermal langerhans cells and induce their apoptosis. Int Immunol 18:879-886

21. Zhao YL, Murthy SN, Manjili MH, Guan LJ, Sen A, Hui SW (2006) Induction of cytotoxic T-lymphocytes by electroporationenhanced needle-free skin immunization. Vaccine 24:1282-1290

22. Colonna M, Trinchieri G, Liu YJ (2004) Plasmacytoid dendritic cells in immunity. Nat Immunol 5:1219-1226

23. Gerlini G, Urso C, Mariotti G, Di Gennaro P, Palli D, Brandani P, Salvadori A, Pimpinelli N, Reali UM, Borgognoni L (2007) Plasmacytoid dendritic cells represent a major dendritic cell subset in sentinel lymph nodes of melanoma patients and accumulate in metastatic nodes. Clin Immunol 125:184-193

24. Charles J, Chaperot L, Salameire D, Di Domizio J, Aspord C, Gressin R, Jacob MC, Richard MJ, Beani JC, Plumas J, Leccia MT (2010) Plasmacytoid dendritic cells and dermatological disorders: focus on their role in autoimmunity and cancer. Eur $\mathbf{J}$ Dermatol 20:16-23

25. Gerlini G, Di Gennaro P, Mariotti G, Urso C, Chiarugi A, Pimpinelli N, Borgognoni L (2010) Indoleamine 2,3-dioxygenase + cells correspond to the BDCA2+ plasmacytoid dendritic cells in human melanoma sentinel nodes. J Invest Dermatol 130:898-901

26. Salio M, Cella M, Vermi W, Facchetti F, Palmowski MJ, Smith CL, Shepherd D, Colonna M, Cerundolo V (2003) Plasmacytoid dendritic cells prime IFN-gamma-secreting melanoma-specific CD8 lymphocytes and are found in primary melanoma lesions. Eur J Immunol 33:1052-1062

27. Gilliet M, Cao W, Liu YJ (2008) Plasmacytoid dendritic cells: sensing nucleic acids in viral infection and autoimmune diseases. Nat Rev Immunol 8:594-606

28. Bantsimba-Malanda C, Marchal-Sommé J, Goven D, Freynet O, Michel L, Crestani B, Soler P (2010) A role for dendritic cells in bleomycin-induced pulmonary fibrosis in mice? Am J Respir Crit Care Med 182:385-395

29. Gabrilovich DI, Chen HL, Girgis KR, Cunningham HT, Meny GM, Nadaf S, Kavanaugh D, Carbone DP (1996) Production of vascular endothelial growth factor by human tumors inhibits the functional maturation of dendritic cells. Nat Med 2:1096-1103

30. Enk AH, Jonuleit H, Saloga J, Knop J (1997) Dendritic cells as mediators of tumour-induced tolerance in metastatic melanoma. Int J Cancer 73:309-316

31. Merad M, Sugie T, Engleman EG, Fong L (2002) In vivo manipulation of dendritic cells to induce therapeutic immunity. Blood 99:1676-1682

32. Stoitzner P, Green LK, Jung JY, Price KM, Tripp CH, Malissen B, Kissenpfennig A, Hermans IF, Ronchese F (2008) Tumour immunotherapy by epicutaneous immunization requires langerhans cells. J Immunol 180:1991-1998

33. Yagi H, Hashizume H, Horibe T, Yoshinari Y, Hata M, Ohshima A, Ito T, Takigawa M, Shibaki A, Shimizu H, Seo N (2006) Induction of therapeutically relevant cytotoxic T lymphocytes in humans by percutaneous peptide immunization. Cancer Res 66:10136-10144 